\title{
Large Bowel Obstruction Caused by Metastatic Prostate Cancer: A Case Report
}

\author{
Mohamad Khaled Almujarkesh ${ }^{1}$ Mahmoud Ismayl ${ }^{2(1)}$ \\ Muhammed Rodwan Hiba ${ }^{5}$ \\ ${ }^{1}$ Gastroenterology and Hepatology Department, University of \\ Damascus, Damascus, Syria \\ 2 Division of Internal Medicine, Creighton University Medical Center, \\ Omaha, Nebraska, United States \\ ${ }^{3}$ Division of Internal Medicine, Sheikh Khalifa Medical City, Abu Dhabi, \\ UAE \\ ${ }^{4}$ Department of Internal Medicine, Division of Digestive Diseases, \\ Rush University Medical Center, Chicago, Illinois, United States \\ ${ }^{5}$ Gastroenterology and Hepatology Department, Oak Hill Hospital, \\ Brooksville, Florida, United States
}

Avicenna J Med 2021;11:217-220.
Ahmad Ismayl ${ }^{3} \quad$ Muhammad Alsayid ${ }^{4}$

Address for correspondence Muhammad Alsayid, MD, MPH, $1725 \mathrm{~W}$ Harrison St, Chicago, IL 60612, United States

(e-mail: mmsayid@gmail.com).

\author{
Abstract \\ Keywords \\ - adenocarcinoma \\ - metastasis \\ - rectal neoplasm \\ - cancer prostate \\ - intestinal obstruction \\ - acute abdomen \\ - case report
}

Bowel obstruction is a frequently encountered condition worldwide that causes numerous admissions to hospitals. Metastatic carcinoma has been identified as one of the infrequently encountered causes of bowel obstruction. Prostate cancer typically metastasizes to lymph nodes, bone, lungs, liver, and brain. In this article, we reported the case of a 75-year-old man who presented with bowel obstruction due to narrowing and stricture of the rectum. Primary rectal mass was initially diagnosed, but upon further investigation, it was found that the mass resembled prostate tissue. The bowel obstruction was managed surgically with a colostomy. The patient was later referred to oncology for chemotherapy and hormonal therapy.

\section{Introduction}

Large bowel obstruction is commonly caused by malignancies in the colon and rectum (60\%), followed by sigmoid volvulus and diverticular disease (30\%). ${ }^{1}$ Prostate cancer is the most frequently diagnosed cancer and the second most common cause of malignancy-related death in men. ${ }^{2} \mathrm{Al}-$ though the course of prostate cancer is usually predictable, on rare occasions, the diagnosis can be challenging. The pattern of metastatic disease of prostate cancer is wellrecognized. Prostate cancer commonly metastasizes to regional lymph nodes and sometimes to bone. ${ }^{3,4}$ However, metastatic prostate cancer to the rectum causing bowel obstruction is extremely rare. Prostate metastasis to the rectum can present with obstructive symptoms similar to those seen in primary carcinoma of the rectum. ${ }^{5}$ Therefore, herein, we reported a case of a patient with metastatic prostate cancer to the rectum causing bowel obstruction.

\section{Case Report}

A 75-year-old African-American man presented to the emergency department with abdominal pain, nausea, vomiting, and abdominal distension of 1-week duration. He denied any passage of stool or flatus for 3 days. Detailed history revealed 2 months of intermittent diffuse abdominal pain associated with constipation and overflow diarrhea. He also complained of fatigue, weight loss, and straining during urination, all of which he attributed to his age, as he was reluctant to seek medical published online November 15, 2021
DOI https://doi.org/ $10.1055 / \mathrm{s}-0041-1736543$. ISSN 2231-0770.

\footnotetext{
(c) 2021. Syrian American Medical Society. All rights reserved. This is an open access article published by Thieme under the terms of the Creative Commons Attribution-NonDerivative-NonCommercial-License, permitting copying and reproduction so long as the original work is given appropriate credit. Contents may not be used for commercial purposes, or adapted, remixed, transformed or built upon. (https://creativecommons.org/ licenses/by-nc-nd/4.0/)

Thieme Medical and Scientific Publishers Pvt. Ltd., A-12, 2nd Floor, Sector 2, Noida-201301 UP, India
} 


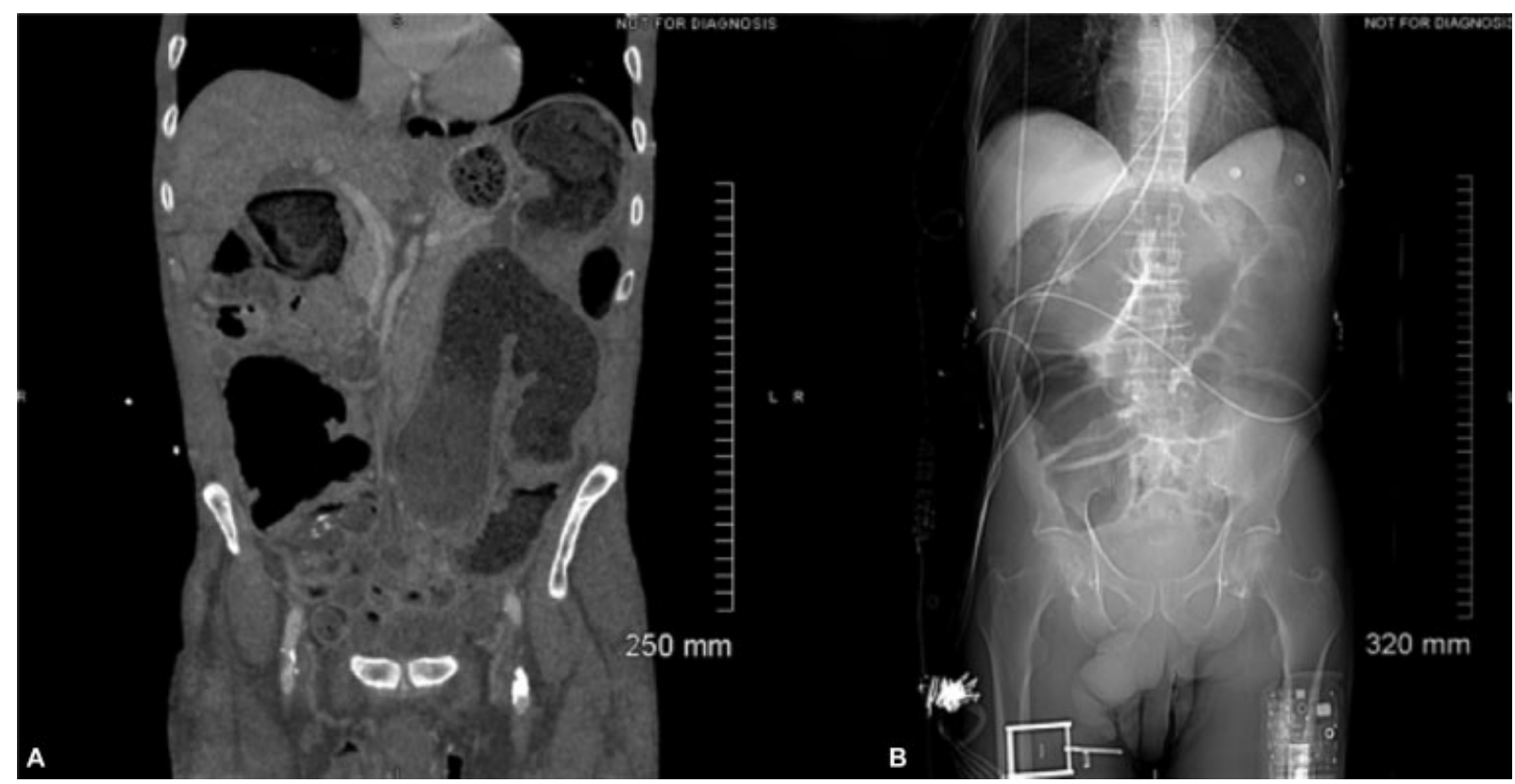

Fig. 1 (A) Computed tomography (CT) scan coronal view showing multiple dilated loops of colon filled with stool and gas. (B) CT scan scout view showing marked dilation of the ascending and transverse colon.

attention. Otherwise, he had no significant past medical or surgical history. On presentation, vital signs were within normal limits. Physical examination showed a distended abdomen with diffuse mild tenderness in the pelvic region without any palpable masses. Digital rectal examination revealed an empty narrow rectum with a hard lumpy mucosal wall all around. The prostate was large and asymmetric with an irregular lumpy surface.

Patient was kept nil per os (NPO) and given intravenous (IV) fluids and morphine. Laboratory tests showed a hemoglobin level of $13.9 \mathrm{~g} / \mathrm{mL}$, hematocrit $40.6 \%$, white blood cell
(WBC) count $3.7 \times 10^{9} / \mathrm{L}$, platelets $161 \times 10^{9} / \mathrm{L}$, total bilirubin $0.6 \mathrm{mg} / \mathrm{dL}$, aspartate aminotransferase $13 \mathrm{U} / \mathrm{L}$, alanine transaminase $18 \mathrm{U} / \mathrm{L}$, lipase $48 \mathrm{U} / \mathrm{L}$, alkaline phosphatase $62 \mathrm{IU} / \mathrm{L}$, and albumin $3.4 \mathrm{~g} / \mathrm{dL}$. Urine analysis was normal. Abdomen and pelvis computed tomography (CT) scans with IV contrast showed colonic dilatation with a large amount of stool throughout the entire colon (-Fig. 1). A circumferential mass surrounding the rectal lumen causing stricture was also observed on magnetic resonance imaging (MRI) of abdomen and pelvis with IV contrast (-Fig. 2). Surgical consultation was done, and a preliminary diagnosis of

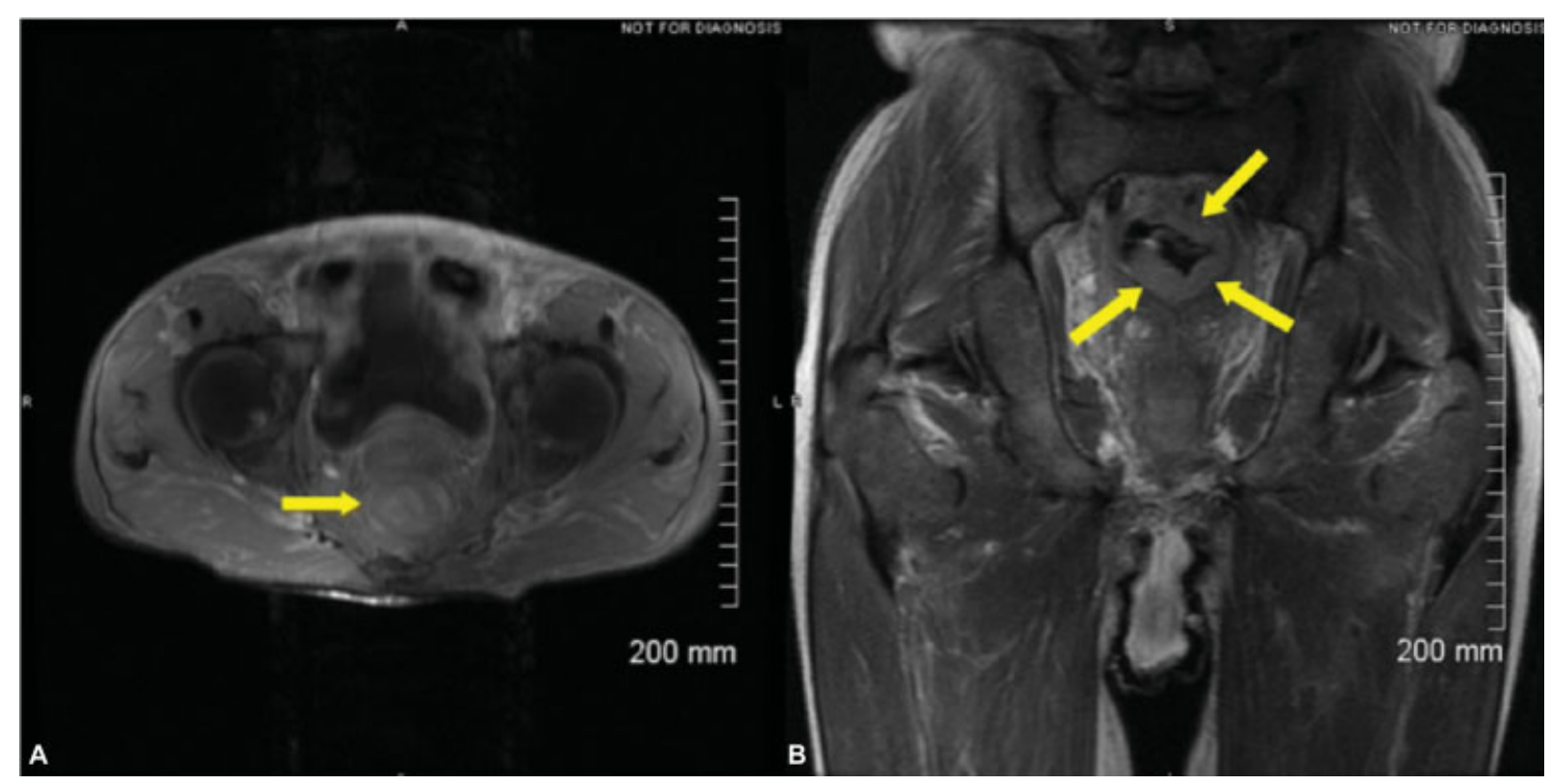

Fig. 2 (A) Magnetic resonance imaging (MRI) with intravenous (IV) contrast axial view showing circumferential enhancing soft tissue, causing narrowing of the rectal lumen (arrow). (B) MRI with IV contrast coronal view showing circumferential enhancing soft tissue, causing narrowing of the rectal lumen (arrows). 


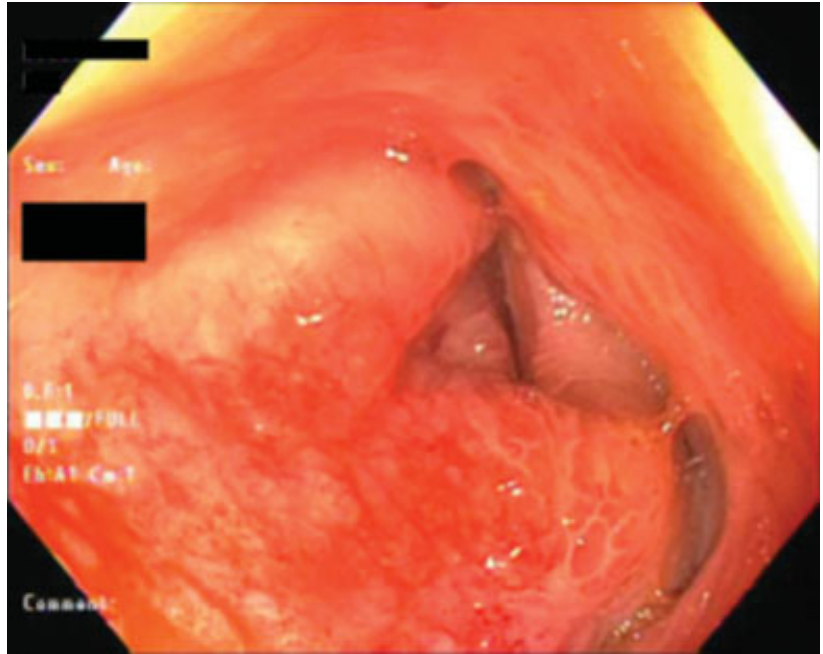

Fig. 3 Flexible sigmoidoscopy showed abnormal mucosa and stenosis.

complete intestinal obstruction due to a primary colorectal malignancy was made. Sigmoidoscopy showed a diffuse area of moderately congested and erythematous mucosa in the distal rectum. An extrinsic severe stenosis measuring $6 \mathrm{~cm}$ (in length) $\times 4 \mathrm{~mm}$ (inner diameter) also was identified via sigmoidoscopy in the distal rectum ( - Fig. 3). A through-thescope dilator was passed and dilation with an $18-20 \mathrm{~mm}$ colonic balloon dilator was performed under fluoroscopic guidance. Biopsies from the stricture were taken with a cold forceps for histology. The biopsies displayed neoplastic glands that are morphologically different from those of the colonic mucosa and similar to the glands of the prostate (-Fig. 4). Under the new suspicion of metastatic prostate cancer, serum prostate specific antigen (PSA) and endoscopic ultrasound-guided core needle biopsy of the rectum were ordered. PSA was elevated $(199 \mathrm{ng} / \mathrm{mL})$ and the endoscopic ultrasound showed diffuse wall thickening of the anal canal and rectum. Biopsies under endoscopic ultrasound guidance showed adenocarcinoma cells, consistent with prostate ori-

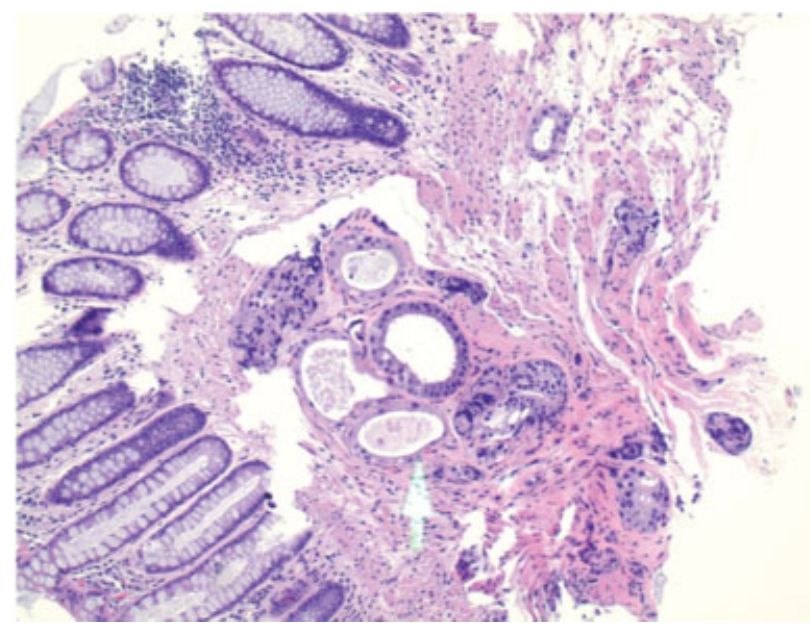

Fig. 4 The rectal biopsy shows neoplastic glands in the submucosa (arrow). In comparison to the overlying mucosa, these glands lack goblet cells and the apical intracytoplasmic mucin. Tumor cells show nuclear hyperchromasia and loss of polarity.

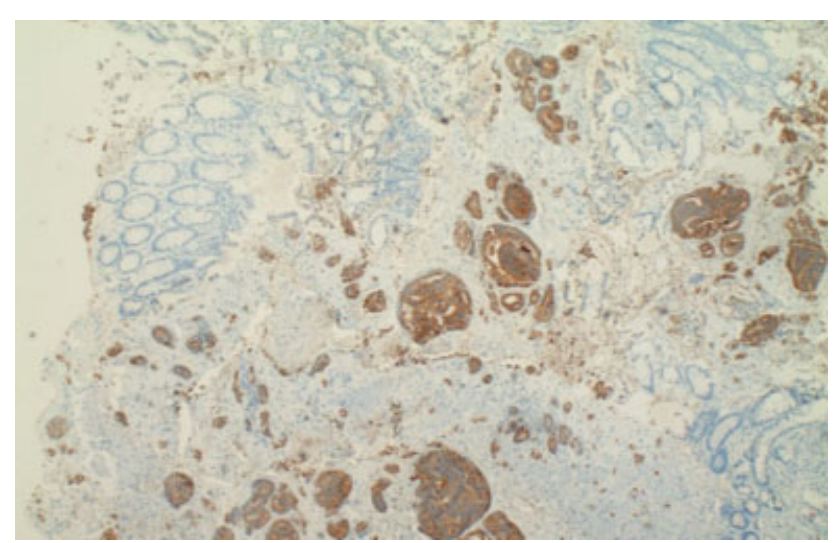

Fig. 5 The infiltrating glands are positive for prostate-specific antigen (PSA) immunostain, while the colorectal mucosa is negative.

gin infiltrating the rectal mucosa (-Fig. 5). The rest of the staging workup was negative.

A final diagnosis of metastatic prostate cancer to the rectum leading to complete intestinal obstruction was made. Therefore, the patient underwent urgent surgical intervention with colostomy to relieve the obstruction. Following this, patient was started on hormone therapy and chemotherapy as part of the long-term management plan.

\section{Discussion}

Prostate cancer occurs in 1 out of 8 men during lifetime in the United States. ${ }^{2}$ Several risk factors have been linked to prostate adenocarcinoma, including family history of the disease, ethnicity, and older age ( $>50$ years). ${ }^{6,7}$ Our patient had two risk factors including his age and race, and mortality due to prostate cancer is higher in African Americans compared with other races in the United States. ${ }^{8}$

During the early stages of the disease, diagnosis can be missed due to the slowly growing nature of the cancer, which explains why our patient presented late to the hospital. Additionally, PSA is subject to false-positive and false-negative results, which affects its clinical efficacy as a standalone test. Therefore, undiagnosed patients usually first present with symptoms of metastasis, just like our patient who did not have any prostate-related symptoms. ${ }^{9,10}$ Infiltration of the rectum is an indicator of late stage prostatic adenocarcinoma, and $38 \%$ of these patients survive beyond 5 years. Patients with prostate cancer and rectal involvement were found to be encountered on average only once every 2 years in a busy colorectal sector. ${ }^{10}$

The prostate gland is separated from the rectal wall by a dense capsule called Denonvilliers' fascia, which consists of a single fibromuscular structure covering the posterior aspect of the prostate and surrounding the seminal vesicles. Direct invasion of the Denonvilliers' fascia is one of three possible routes that have been identified for prostate cancer to infiltrate the colorectal tissue. ${ }^{6}$ The second is via pelvic lymph nodes that receive drainage from the prostate gland and the rectum. Third route is by seeding of cancer cells into the perirectal or rectal tissue during prostate biopsy. ${ }^{6}$ 
220 A Rare Cause of Large Bowel Obstruction Almujarkesh et al.

\section{Conclusion}

Although rectal infiltration by prostatic adenocarcinoma is a rare presentation of prostate cancer, physicians should consider prostate cancer metastasis as a differential diagnosis for carcinomas found in the rectal wall in men. In addition, PSA immunohistochemical staining is a sensitive and specific marker of prostatic differentiation and should be used in doubtful cases, even in the absence of urinary symptoms.

\section{Informed Consent}

A written informed consent was obtained from the patient for presentation of this case.

\section{Funding}

M.A. was funded by the National Center For Advancing Translational Sciences of the National Institutes of Health under Award number TL1TR002388. The content is solely the responsibility of the authors and does not necessarily represent the official views of the National Institutes of Health

The rest of authors received no financial support for the research, authorship, and/or publication of this article.

\section{Conflict of Interest}

None declared.

\section{References}

1 Catena F, De Simone B, Coccolini F, Di Saverio S, Sartelli M, Ansaloni L. Bowel obstruction: a narrative review for all physicians. World J Emerg Surg 2019;14:20

2 Siegel RL, Miller KD, Fuchs HE, Jemal A. Cancer Statistics, 2021. CA Cancer J Clin 2021;71(01):7-33

3 Venara A, Thibaudeau E, Lebdai S, et al. Rectal metastasis of prostate cancer: about a case. J Clin Med Res 2010;2(03):137-139

4 Bubendorf L, Schöpfer A, Wagner U, et al. Metastatic patterns of prostate cancer: an autopsy study of 1,589 patients. Hum Pathol 2000;31(05):578-583

5 Bowrey DJ, Otter MI, Billings PJ. Rectal infiltration by prostatic adenocarcinoma: report on six patients and review of the literature. Ann R Coll Surg Engl 2003;85(06):382-385

6 Tang T, Yang Z, Zhang D, Qu J, Liu G, Zhang S. Clinicopathological study of 9 cases of prostate cancer involving the rectal wall. Diagn Pathol 2017;12(01):8

7 National Cancer Institute. Physician Data Query (PDQ). Prostate Cancer Prevention. Available at: March 21, 2019 at https://www. cancer.gov/types/prostate/hp/prostate-prevention-pdq

8 Giona S. The epidemiology of prostate cancer. In: Bott SRJ, Ng KL, eds. Prostate Cancer. Accessed September 27, 2021 at: https:// www.ncbi.nlm.nih.gov/books/NBK571326/doi:10.36255/exonpublications.prostatecancer.epidemiology.2021

9 Abbas TO, Al-Naimi AR, Yakoob RA, Al-Bozom IA, Alobaidly AM. Prostate cancer metastases to the rectum: a case report. World J Surg Oncol 2011;9:56

10 Wang H, Yao Y, Li B. Factors associated with the survival of prostate cancer patients with rectal involvement. Diagn Pathol 2014;9:35 